\title{
Doing Our Part to Mitigate the Opioid Crisis
}

\author{
Crystal Fancher, MD, and Alicia M. Terando, MD \\ Division of Breast, Endocrine and Soft Tissue Surgery, Department of Surgery, Keck School of Medicine of USC, Los \\ Angeles, CA
}

In 2016, there were a reported 42,000 opioid-related deaths in the US, $40 \%$ of which involved prescription opioids. The Centers for Disease Control and Prevention (CDC) reports that from 1999 to 2017 there were over 700,000 drug overdoses and, in 2017 alone, 70,000 people died from drug overdose, with $68 \%$ of those deaths attributed to prescription or illicit opioid use ${ }^{1}$. While the opioid epidemic was only officially declared to be a public health emergency by the US Department of Health and Human Services in 2017, the factors bringing our society to this point are multiple and go back several decades. One issue was the underestimation of the addictive potential of opioid medications. In 1980, The New England Journal of Medicine published a one-paragraph Letter to the Editor in which the authors reported their observation of 12,000 hospitalized patients who received 'at least one narcotic preparation', and found that only four patients became addicted. This led to the erroneous conclusion that it is rare for patients treated with narcotics to become addicted, a principle that soon became $\operatorname{dogma}{ }^{2}$. This misinformation persisted, as evidenced in a 2001 monograph published by the National Pharmaceutical Council and the Joint Commission on Accreditation of Healthcare Organizations (JCAHO) in which they state, "In general, patients in pain do not become addicted to opioids. Although the actual risk of addiction is unknown, it is thought to be quite low." 3

In a 1990 editorial in Annals of Internal Medicine, Dr. Mitchell Max, then President of the American Pain Society, wrote of the failure of the medical community to

(C) Society of Surgical Oncology 2019

First Received: 17 November 2019;

Published Online: 18 December 2019

A. M. Terando, MD

e-mail: alicia.terando@med.usc.edu properly assess and manage pain ${ }^{4}$. This was a laudable and effective call to action, raising the awareness of the medical community regarding its systematic undertreatment of pain, and ultimately leading to JCAHO's institution of standards for pain assessment and measurement, and the US Veterans Health Administration's publication of 'Pain: The Fifth Vital Sign', the manifestation of which we see every day in the form of bedside pain scores ${ }^{5}$. However, while the intent of these measures was to relieve suffering, the unintended consequence is that our society has now come to think of pain as something that is universally bad that needs to be eliminated, rather than accepted as a natural part of life. In an opinion piece published in the New York Times, Firoozeh Dumas illustrated the stark difference in the American versus European culture with regard to the perception and management of pain, as she wrote about her experience as an American living in Germany and undergoing a hysterectomy. She describes asking her gynecologist, surgeon, and anesthesiologist in turn about postoperative pain management, and her shock to be informed by each of them that narcotic pain medication would not be necessary. In fact, she was told that feeling some discomfort is not only a fact of life but that she should think of it as her body's signal as to how she was healing, to keep her from overexertion, and to let her know when to rest. In the end, she did just fine without narcotics ${ }^{6}$.

It is easy to see how the perception of pain as an entity to be eradicated, combined with a sense of obligation on the part of prescribers to keep patients pain-free, the normalization of the provision and receipt of narcotic prescriptions postoperatively, and a regulatory system that makes it impractical to provide more opioids to patients who may have not been prescribed enough, have conspired to bring us to our current state of crisis. There are undoubtedly many other inextricable social and economic factors that have led us here, but the question now becomes 'how can surgeons contribute to the slowing and ultimate 
reversal of this problem?' The most direct manner in which surgeons can impact the number of opioids available for misuse or diversion is through their prescribing practices. Until recently, there has been little to no data to guide surgeons as to how many narcotic pills to prescribe for any given patient or clinical scenario, leading to wide variations in practice. In an effort to help guide prescribing practices, in this issue of Annals of Surgical Oncology Park et al. ${ }^{7}$ report their exploration of the patient characteristics and surgical factors associated with increased opioid requirements following breast-conserving surgery.

Through analysis of the results of a telephone survey taken of 316 patients at postoperative days 7-10 following breast-conserving surgery, they found that preoperative narcotic use, younger age, current smoking, and high postoperative pain scores were factors associated with higher postoperative opioid use. Surgical factors including the extent of axillary surgery, bilateral oncoplastic surgery, placement of at least one surgical drain, and receiving a discharge prescription of $>150$ oral morphine equivalents (OMEs) were also associated with high postoperative opioid use. Interestingly, they note that those who received more pills used more pills, and those who received less used less. This suggests the possibility of a psychological component-if a patient is prescribed more narcotics, does that somehow lead the patient to believe that they should be expecting a lot of pain, and vice versa? Furthermore, Park et al. ${ }^{7}$ observed that for many, mean opioid use was less than prescribed. Similar findings were seen in a study of opioid use following secondary reconstruction published by Hart et al., in which the authors reported that while prescribing 30 opioid pills provided patients with enough medication, there were many pills left unused. They also found that patients stopped using opioids by postoperative day 5 , with an average of 18.6 excess tablets ${ }^{8}$. As would be expected, Park et al. ${ }^{7}$ report that opioid consumption is correlated with the extent/invasiveness of the surgical procedure. While it may seem obvious that the amount of narcotic prescribed would be proportional to the extent of surgery performed, a retrospective study by Murphy et al. ${ }^{9}$ showed that in patients undergoing mastectomy, although patient-reported pain varied by procedure, prescribing practices did not reflect this difference.

With 500,000 breast operations occurring each year in the US, there is a significant opportunity for breast surgeons to make a positive impact on the opioid crisis. By tailoring opioid prescriptions to patient characteristics and extent of surgery, we can attempt to optimize the number of opioids prescribed for each patient, and minimize misuse and diversion. While this will not solve the opioid epidemic single-handedly, it is certainly a step each of us can take to do our part to mitigate the opioid crisis. Going forward, knowing that, as Park et al. $^{7}$ also reported, a substantial proportion of patients do not require any narcotics at all following breast-conserving surgery, we may begin to make changes in how we counsel patients as to their expectations regarding postoperative opioids, and perhaps begin to change our own culture with respect to prescribing practices. With a concerted effort towards change in practice and expectations, prescribing opioids following surgery may ultimately become the exception rather than the rule.

DISCLOSURE Crystal Fancher and Alicia M. Terando declare no conflict of interest.

\section{REFERENCES}

1. Centers for Disease Control and Prevention. Opioid overdose. Available at: https://www.cdc.gov/drugoverdose. Accessed 11 November 2019.

2. Porter J, Jick H. Addiction rare in patients treated with narcotics. $N$ Engl J Med. 1980; 302(2):123.

3. National Pharmaceutical Council, Inc., Joint commission on accreditation of healthcare organizations. Pain: current understanding of assessment, management, and treatments. Available at: https://www.npcnow.org/system/files/research/download/Pain-Cur rent-Understanding-of-Assessment-Management-and-Treatments. pdf. Accessed 11 November 2019.

4. Baker DW. The joint commission's pain standards: origins and evolution. Oakbrook Terrace, IL: The Joint Commission; 2017.

5. Department of Veteran Affairs. Pain: the fifth vital sign. Washington, DC: Department of Veteran Affairs; 2000.

6. Dumas F. After surgery in Germany, I wanted vicodin, not herbal tea. New York, NY: New York Times; 2017

7. Park KU, Kyrish K, Yi M. Opioid use after breast-conserving surgery: prospective evaluation of risk factors for high opioid use. Ann Surg Oncol. (in press)

8. Hart AM, Broecker JS, Kao L, Losken A. Opioid use following outpatient breast surgery: are physicians part of the problem? Plast Reconstr Surg. 2018; 142(3):611-620.

9. Murphy BL, Thiels CA, Hanson KT, et al. Pain and opioid prescriptions vary by procedure after breast surgery. J Surg Oncol. 2019; 120(4):593-602.

Publisher's Note Springer Nature remains neutral with regard to jurisdictional claims in published maps and institutional affiliations. 